\title{
Utilizing preprocedural CT scans to identify patients at risk for suboptimal external ventricular drain placement with the freehand insertion technique
}

\author{
Mitchell P. Wilson, MD, ${ }^{1}$ Cian O'Kelly, MD, MSc, FRCSC, ${ }^{2}$ Andrew S. Jack, MD, MSc, ${ }^{2}$ and \\ Jeremy Rempel, MD, DABR, FRCPC ${ }^{1}$
}

\begin{abstract}
'Department of Radiology and Diagnostic Imaging, and 2Division of Neurosurgery, Department of Surgery, University of Alberta,
\end{abstract} Edmonton, Alberta, Canada

\begin{abstract}
OBJECTIVE Freehand insertion of external ventricular drains (EVDs) using anatomical landmarks is considered the primary method for placement, although alternative techniques have shown improved accuracy in positioning. The purpose of this study was to retrospectively evaluate which features of the baseline clinical history and preprocedural CT scan predict EVD positioning into suboptimal and unsatisfactory locations when using the freehand insertion technique.

METHODS A retrospective chart review was performed evaluating 189 consecutive adult patients who received an EVD via freehand technique through an anterior burr hole between January 1, 2014, and December 31, 2015, at a Level 1 trauma facility in Edmonton, Alberta, Canada. The primary outcome measures included features associated with suboptimal positioning (Kakarla grade 1 vs Kakarla grades 2 and 3 ). The secondary outcome measures were features associated with unsatisfactory positioning (Kakarla grades 1 and 2 vs Kakarla grade 3 ).

RESULTS Fifty-one EVDs (27\%) were suboptimally positioned. Fifteen (8\%) EVDs were placed into eloquent cortex or nontarget CSF spaces. Admitting diagnosis, head height-to-width ratio in axial plane, and side of predominant pathology were found to be significantly associated with suboptimal placement $(p=0.02,0.012$, and 0.02 , respectively). A decreased height-to-width ratio was also associated with placement into only eloquent cortex and/or nontarget CSF spaces $(p=0.003)$.
\end{abstract}

CONCLUSIONS Freehand insertion of an EVD is associated with significant suboptimal positioning into parenchyma and nontarget CSF spaces. The likelihood of inaccurate EVD placement can be predicted with baseline clinical and radiographic features. The patient's height-to-width ratio represents a novel potential radiographic predictor for malpositioning. https://thejns.org/doi/abs/10.3171/2018.1.JNS172839

KEYWORDS trauma; hydrocephalus; surgical technique; freehand; external ventricular drain

$\mathrm{F}$ REEHAND insertion of external ventricular drains (EVDs) is one of the most common lifesaving neurosurgical procedures. The procedure is often performed at the patient's bedside using anatomical landmarks to determine catheter trajectory. Though generally considered a safe procedure, concerns have been raised regarding the accuracy of placement with the freehand technique. ${ }^{17,22}$ While serious complications of malpositioning are rare in the literature, these complications may be underreported. ${ }^{17}$ Malpositioning can also result in less se- vere complications that are not typically evaluated, such as earlier catheter obstruction and subtle long-term cognitive changes, even when placement is in suboptimal functional locations. , $^{318,22}$ Difficulties in placement with multiple passes may also lead to an increased risk of subclinical hemorrhages, which can result in seizures, and can serve as a contraindication to anticoagulation. ${ }^{3}$

Since the advent of the Ghajar Guide, a fixating tripod to assist in guiding placement, an increasing number of promising alternatives to freehand insertion have been de-

ABBREVIATIONS EVD = external ventricular drain; GCS = Glasgow Coma Scale; ICU = intensive care unit; PACS = picture archiving and communication system. SUBMITTED November 13, 2017. ACCEPTED January 4, 2018. 
veloped $2,3,5,10,12,15,20,22$ that have been shown to increase the accuracy of placement. 4,6,11,13,15,20,21,24 However, alternatives to freehand insertion have inherent limitations, some of which can be particularly restrictive in the emergency setting. Stratifying patients at risk for malpositioning with the freehand technique based on clinical and radiographic characteristics can help identify those patients most likely to benefit from an alternative EVD insertion technique.

Previous studies have associated small ventricle size, ${ }^{7,16,23}$ decreased Evan's index, ${ }^{7}$ and degree of midline shift ${ }^{9}$ with malpositioning. A comprehensive evaluation of the preprocedural CT scan for predictors of malpositioning has not been reported. The purpose of this paper was to evaluate whether baseline clinical and/or radiographic features performed prior to drain insertion can predict malpositioning of EVDs when using the freehand insertion technique.

\section{Methods \\ Study Population}

Local institutional approval was obtained from the University of Alberta Health Research Ethics Board (Pro00064177). A retrospective chart review of all patients admitted to the University of Alberta Hospital Neuroscience Intensive Care Unit (ICU) in Edmonton, Alberta, Canada, between January 1, 2014, and December 31,2015 , was performed to identify all adult patients receiving an EVD during that time. EVDs inserted through frontal bone burr holes with the freehand insertion technique were selected for review. All EVDs were inserted by the neurosurgical team, which included residents, neurosurgeons, and neurointensivists. EVDs were inserted to monitor and/or reduce intracranial pressure, or to provide temporary CSF drainage in patients with infected CSF shunts.

\section{Data Collection}

Patients were evaluated via our institution's picture archiving and communication system (PACS) to assess both preinsertion and postinsertion CT scans. Local CT head protocol includes axial acquisition in 5-mm slices from vertex to skull base. Patients without both a preinsertion and postinsertion CT scan, and patients with an EVD inserted via a posterior approach, were excluded from analysis. Demographic information collected included age, sex, diagnosis, admitting Glasgow Coma Scale (GCS) score, and hospital location of EVD insertion. Preinsertion CT scans were reviewed for thickness of superficial soft tissue at approximately Kocher's point (1-2 cm anterior to the coronal suture in the midpupillary line); skull thickness at Kocher's point; distance from the inner table of the frontal bone at Kocher's point to the ipsilateral anterior horn; ratio of the inner table anteroposterior to transverse dimensions at the maximal transverse skull diameter in corrected axial series (height-to-width ratio); ratio of maximum width of the lateral ventricular frontal horns to the maximal transverse internal diameter of the skull at the same level (Evan's index); ratio of the unilateral ventricle size to the unilateral hemisphere width from midline at the same level (unilateral ventricle ratio); amount of maxi-
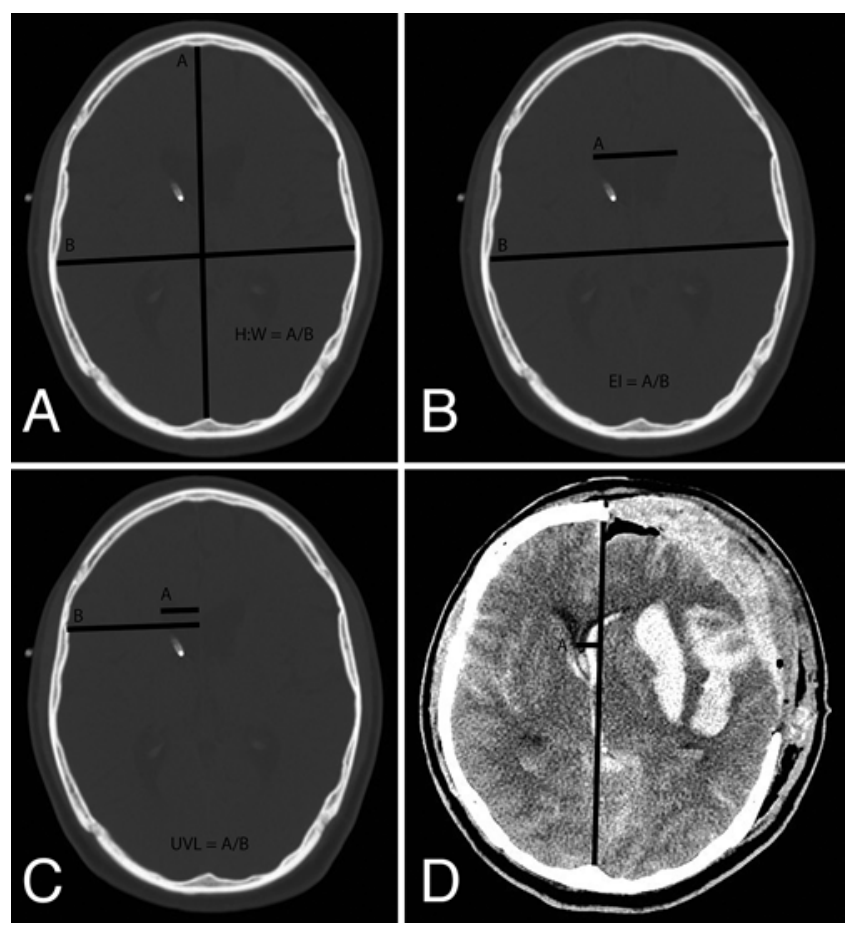

FIG. 1. Axial CT examples of radiographic measurements: height-towidth ratio $(\mathbf{A})$, Evan's index $(\mathbf{B})$, unilateral ventricle ratio $(\mathbf{C})$, and midline shift (D).

mal midline shift; presence of other pathology noted on CT (including bleed, craniotomy, tumor, additional prior shunt, and other); type of other pathology present, including the number of different types of intracranial bleeds; and laterality of the pathology (ipsilateral, contralateral, or both). Measurements for height-to-width ratio, Evan's index, unilateral ventricle ratio, and midline shift are demonstrated in Fig. 1. Postinsertion CT scans were reviewed for side of drain insertion and the Kakarla grade (Kakarla). Data were extracted by one author. The author was not involved in the direct management and/or EVD placement of any patient included in this study. The primary outcome measures included features associated with suboptimal positioning (Kakarla grade 1 vs Kakarla grades 2 and 3). The secondary outcome measures were features associated with unsatisfactory positioning (Kakarla grades 1 and 2 vs Kakarla grade 3 ).

The Kakarla grading system for catheter tip insertion stratifies the accuracy of tip placement into 3 categories based on 1) optimal placement within the ipsilateral frontal horn or third ventricle through the foramen of Monro; 2) suboptimal functional placement in the contralateral lateral ventricle or noneloquent cortex; and 3) unsatisfactory placement into eloquent cortex or nontarget CSF space, with or without functional drainage (Fig. 2). ${ }^{9}$ The grading system has previously demonstrated almost perfect intraobserver variability and strong interobserver variability (kappa $=0.922$ and 0.846 , respectively). ${ }^{9}$

\section{Statistical Analysis}

Demographics and clinical characteristics of the pa- 

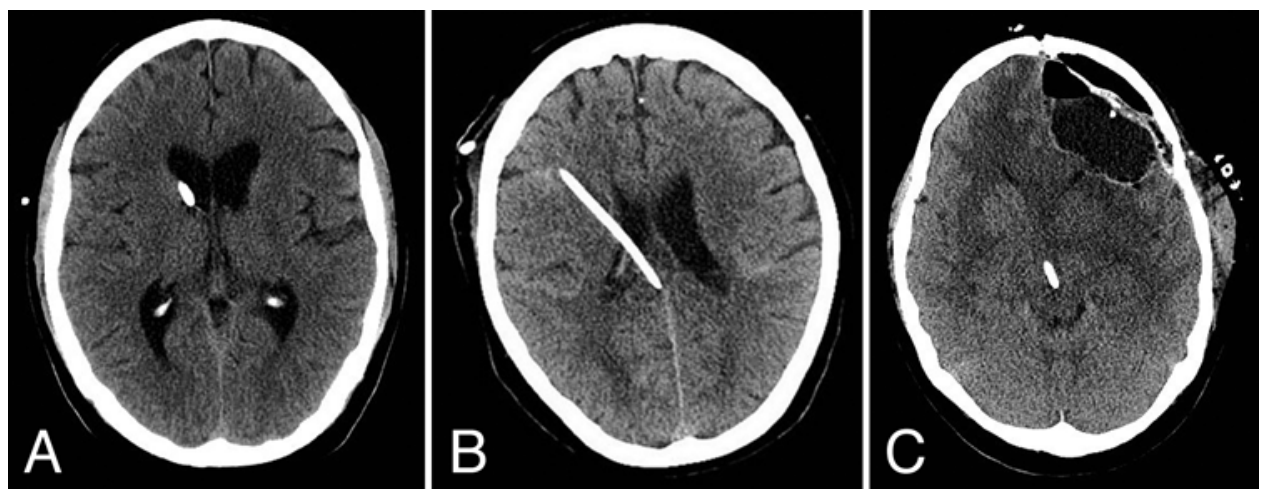

FIG. 2. Axial CT examples of Kakarla grade 1 placement with EVD tip placed in the ipsilateral frontal horn (A), Kakarla grade 2 suboptimal placement with the tip in noneloquent cortex $(B)$, and Kakarla grade 3 unsatisfactory placement with the tip positioned in the midbrain (C).

tients were described using mean for continuous variables and median for ordinal variables. A t-test was performed to compare the patients' characteristics between Kakarla grades. Categorical variables were compared using the chisquare test. However, when the validity of the chi-square test was uncertain due to small cell counts, we used Fisher's exact test. Logistic regression analysis was used for categorical variables with more than two levels. Statistical analyses were conducted in SAS (version 9.4, SAS Institute Inc.).

\section{Results}

A total of 246 EVD insertion procedures occurred during the 2-year period, of which 48 patients received the EVD intraoperatively through a craniotomy site. Five additional patients were excluded from analysis as they died prior to receiving a postinsertion CT scan, and 4 patients were excluded due to posterior insertion of the EVD. A total of 189 freehand EVD insertion procedures were included in the analysis. One hundred thirty-eight patients (73\%) were Kakarla grade 1, $36(19 \%)$ were Kakarla grade 2, and $15(8 \%)$ were Kakarla grade 3 (Table 1). In patients with suboptimal placement, a statistically significant difference in presenting diagnosis was present $(\mathrm{p}=0.02$; Table 2). A global test for significance demonstrated an increased rate of vascular pathologies in patients with optimal EVD positioning. Age was the only significant difference identified in baseline clinical characteristics for patients with unsatisfactory EVD placement $(p=0.02)$, which may be related to an imbalance of other predictors with differing ages in these groups (Table 3 ).

Patients with suboptimal EVD positioning were more likely to have a smaller height-to-width ratio (1.22 vs 1.25 , $p=0.012$ ). The difference was more pronounced when assessing for unsatisfactory EVD positioning (1.19 vs 1.25, $p=0.003$ ). Side of the predominant additional pathology demonstrated a significant difference in suboptimal positioning $(p=0.02)$, with global test for significance revealing differences in ipsilateral and bilateral features between groups as the cause for differences. Side of predominant feature was not found to represent a significant difference in unsatisfactory placement $(\mathrm{p}=0.55)$. No differences were identified for soft tissue diameter, skull thickness, distance from inner table to frontal horn, Evan's index, unilateral ventricle ratio, degree of midline shift, number of different features, or number of different intracranial bleeds in either suboptimal or unsatisfactory placements. An example of a Kakarla grade 3 patient is demonstrated in Fig. 3.

TABLE 1. Baseline patient demographics

\begin{tabular}{|c|c|}
\hline Variable & Value (\%) \\
\hline No. of patients & 189 \\
\hline Mean age (yrs) & 53 \\
\hline \multicolumn{2}{|l|}{ Sex } \\
\hline Male & $93(49)$ \\
\hline Female & $96(51)$ \\
\hline \multicolumn{2}{|l|}{ Diagnosis } \\
\hline Neoplasm & $38(21)$ \\
\hline Trauma & $24(13)$ \\
\hline Vascular & $117(63)$ \\
\hline Other* & $6(3)$ \\
\hline Median admitting GCS score & 10 \\
\hline Mean ICU LOS (days) & 11 \\
\hline \multicolumn{2}{|l|}{ ICU outcome } \\
\hline Survived & $157(83)$ \\
\hline Died & $32(17)$ \\
\hline \multicolumn{2}{|l|}{ Location of EVD insertion } \\
\hline ER & $29(16)$ \\
\hline $\mathrm{NICU}$ & $102(58)$ \\
\hline OR & $46(26)$ \\
\hline Missing $†$ & 12 \\
\hline \multicolumn{2}{|l|}{ Position of insertion } \\
\hline Rt frontal & $170(90)$ \\
\hline Lt frontal & $19(10)$ \\
\hline \multicolumn{2}{|l|}{ Kakarla grade } \\
\hline 1-optimal & $138(73)$ \\
\hline 2-suboptimal & $36(19)$ \\
\hline 3-unsatisfactory & $15(8)$ \\
\hline
\end{tabular}

ER = emergency room; LOS = length of stay; NICU = neurointensive ICU; OR $=$ operating room.

* Other = congenital, infection, demyelination, toxic, metabolic, degenerative. $\dagger$ Denotes information not available in the NICU database. 
TABLE 2. Assessing clinical and radiographic characteristics in suboptimal EVD placement

\begin{tabular}{|c|c|c|c|}
\hline \multirow[b]{2}{*}{ Characteristic } & \multicolumn{2}{|c|}{ Kakarla Grade (\%) } & \multirow{2}{*}{$\begin{array}{c}\mathrm{p} \\
\text { Value }\end{array}$} \\
\hline & 1 & $2 \& 3$ & \\
\hline No. of patients & 138 & 51 & \\
\hline Mean age (yrs) & 53 & 51 & 0.37 \\
\hline Males & $69(50)$ & $24(47)$ & 0.72 \\
\hline Diagnosis & & & 0.02 \\
\hline Neoplasm & $21(16)$ & $17(33)$ & \\
\hline Trauma & $15(11)$ & $9(18)$ & \\
\hline Vascular* & $94(70)$ & $23(45)$ & \\
\hline Other & $4(3)$ & $2(4)$ & \\
\hline Missing $†$ & 4 & 0 & \\
\hline Mean admitting GCS score & 9 & 9 & 0.96 \\
\hline Soft tissue diameter (cm) & 0.46 & 0.49 & 0.39 \\
\hline Skull thickness (cm) & 0.76 & 0.74 & 0.85 \\
\hline Brain depth to ventricle $(\mathrm{cm})$ & 3.36 & 3.32 & 0.65 \\
\hline Head height-to-width ratio & 1.25 & 1.22 & 0.012 \\
\hline Evan's index & 0.31 & 0.29 & 0.21 \\
\hline Unilateral ventricle ratio & 0.34 & 0.35 & 0.53 \\
\hline Side of predominant pathology & & & 0.02 \\
\hline |psilateral ${ }^{*}$ & $8(6)$ & $8(16)$ & \\
\hline Contralateral & $26(19)$ & $14(27)$ & \\
\hline Bilateral $^{*}$ & $65(47)$ & $13(25)$ & \\
\hline None & $39(28)$ & $16(31)$ & \\
\hline Midline shift (cm) & -0.1 & -0.1 & 0.57 \\
\hline
\end{tabular}

Boldface type indicates statistical significance.

* Global test for significance identifies this value as a cause for differences between groups.

† Denotes information not available in the NICU database.

\section{Discussion}

Ventriculostomies were first described in the 1700s, with the current freehand technique remaining relatively unchanged since the 1950s. ${ }^{19}$ Freehand insertion remains the sole means of catheter placement by more than $90 \%$ of neurosurgeons. ${ }^{14}$ This technique is often performed at bedside, using surface anatomical landmarks to determine the trajectory of the catheter. This technique is generally considered safe, although concerns have been raised regarding the accuracy of EVD placement, ${ }^{17,22}$ with advocates for alternative placement techniques citing the accuracy rates as unacceptably low. ${ }^{1}$

\section{Malposition Rates}

Several studies have assessed the rate of malpositioning, citing malposition rates between $2 \%$ and $60 \%$, $7-9,18,22,25$ with an average of 1.9 passes per placement. ${ }^{16}$ Lower rates of malpositioning were cited in studies defining malpositioning only when the distal tip was placed within brain parenchyma or extraventricular spaces. ${ }^{18,25}$ When a broader definition of malpositioning was used, limiting acceptable placement to include only optimal positioning within the ipsilateral frontal horn or third ventricle through the frontal horn, higher rates of malpositioning ranging from $23 \%$ to $60 \%$ were identified. ${ }^{7-9,22}$ Our study identified rates
TABLE 3. Assessing clinical and radiographic characteristics in unsatisfactory EVD placement

\begin{tabular}{|c|c|c|c|}
\hline \multirow[b]{2}{*}{ Characteristic } & \multicolumn{2}{|c|}{ Kakarla Grade (\%) } & \multirow{2}{*}{$\begin{array}{c}p \\
\text { Value }\end{array}$} \\
\hline & $1 \& 2$ & 3 & \\
\hline No. of patients & 174 & 15 & \\
\hline Mean age (yrs) & 53 & 45 & 0.02 \\
\hline Males & $88(51)$ & $5(33)$ & 0.20 \\
\hline Diagnosis & & & 0.72 \\
\hline Neoplasm & $34(20)$ & $4(27)$ & \\
\hline Trauma & $22(13)$ & $2(13)$ & \\
\hline Vascular & $108(64)$ & $9(60)$ & \\
\hline Other & $6(4)$ & $0(0)$ & \\
\hline Missing* & 4 & 0 & \\
\hline Mean admitting GCS score & 10 & 7 & 0.07 \\
\hline Soft tissue diameter (cm) & 0.47 & 0.44 & 0.32 \\
\hline Skull thickness (cm) & 0.75 & 0.77 & 0.80 \\
\hline Brain depth to ventricle $(\mathrm{cm})$ & 3.34 & 3.45 & 0.48 \\
\hline Head height-to-width ratio & 1.25 & 1.19 & 0.003 \\
\hline Evan's index & 0.31 & 0.27 & 0.06 \\
\hline Unilateral ventricle ratio & 0.34 & 0.35 & 0.76 \\
\hline Side of predominant pathology & & & 0.55 \\
\hline Ipsilateral & $14(8)$ & $2(13)$ & \\
\hline Contralateral & $35(20)$ & $5(33)$ & \\
\hline Bilateral & $73(42)$ & $5(33)$ & \\
\hline None & $52(30)$ & $3(20)$ & \\
\hline Midline shift (cm) & -0.1 & -0.1 & 0.85 \\
\hline
\end{tabular}

of malpositioning in accordance with these published rates, with a suboptimal malposition rate of $27 \%$, and $8 \%$ within unsatisfactory positions, including eloquent cortex and nontarget CSF spaces.

While many clinicians accept functional ventricular placement as adequate, Toma et al. have shown that malpositioning even just posterior to the foramen of Monro can be associated with significantly increased rates of proximal catheter failure. ${ }^{22}$ The proposed mechanism of obstruction is related to the presence of choroid plexus posterior to the level of the foramen of Monro. Other potential complications of freehand insertion, including high rates of hemorrhages and long-term cognitive changes, have been proposed but poorly evaluated., ${ }^{3,18,22}$ Fiorella et al., for example, have argued that malposition rates and potential for increased required number of passes can result in higher risks for small hemorrhages. They propose that even when these hemorrhages are small and not initially clinically significant, they can still result in an inciting focus for future seizures, and can serve as a contraindication to anticoagulation. While other more serious acute complications of EVD malpositioning are rarely reported, underreporting has been speculated. ${ }^{17}$ In light of the potential risk for complications even in suboptimal functional locations, we felt a definition of malpositioning assessing for optimal placement was most appropriate for primary analysis. 


\section{Predictors for Malpositioning}

In a series of 129 EVD insertions, Hsieh et al. noted a significantly higher risk of malpositioning in the presence of smaller ventricular ratios (Evan's index), and smaller

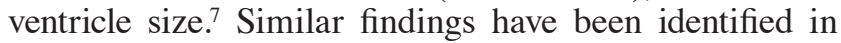
which an Evan's index $<0.3$ in patients was significantly more likely to result in malpositioning than when the ratio was $>0.4 .{ }^{22}$ Other studies have found that smaller ventricular sizes and a decreased frontal occipital horn width ratio were associated with increased rates of malpositioning, ${ }^{23}$ and note an increased number of passes required for successful cannulation in patients with smaller ventricular volumes..$^{16}$ Our study did not corroborate an association of relative ventricle size with rates of malpositioning, although a smaller Evan's index ratio did approach significance $(0.27$ vs $0.31, p=0.06)$ in assessment of unsatisfactory positioning. Furthermore, age was identified as a significant factor associated with unsatisfactory positioning (53 vs 45 years old, $p=0.02$ ), which may correlate with ventricle size, in which younger patients who are more likely to have smaller ventricles are more difficult to catheterize. Taken together, our findings in combination with previous studies in the literature may suggest that relative ventricle size is important for successfully accessing the ventricle, but not in ensuring optimal placement, which requires greater accuracy during the initial trajectory.

Mixed evidence is observed in the presence of midline shift. In the largest published series of 521 ventriculostomies, Kakarla et al. noted a trend toward a significant risk of malpositioning in the presence of midline shift $(\mathrm{p}$ $=0.059$ ) and when the drain was inserted contralateral to the midline shift $(\mathrm{p}=0.063) .{ }^{9}$ These findings have not been corroborated in smaller series. Our study also did not identify an increased risk of malpositioning in this group. It is possible that increased rates of malpositioning occur in a subset of patients with more pronounced midline shift, but our study did not have the power to perform this subgroup analysis. We did note an increased risk of suboptimal placement when a predominant intracranial pathology was unilateral, which could result in a shift in the relative lateral ventricle position, even in the absence of significant midline shift. These observations may correlate with previous findings suggesting an increased number of passes required when a midline hematoma was present ${ }^{16}$ and trend toward significance for midline shift ${ }^{9}$ as indirect indicators that altering the expected lateral ventricle position relative to external landmarks results in increased difficulty with blind freehand placement. This could account for why midline shift was not significant in our study, where the averages in both groups were relatively low, and unlikely to shift the relative position of the lateral ventricles. This may also account for why a relatively higher proportion of patients with suboptimal EVD position tended to have a unilateral predominant abnormality as opposed to a higher proportion of optimally positioned patients having bilateral pathology. Furthermore, this would account for a higher proportion of optimally positioned patients having vascular pathologies, which predominantly represented unilateral and symmetric subarachnoid hemorrhages.

Perhaps most importantly, our study identified signifi-
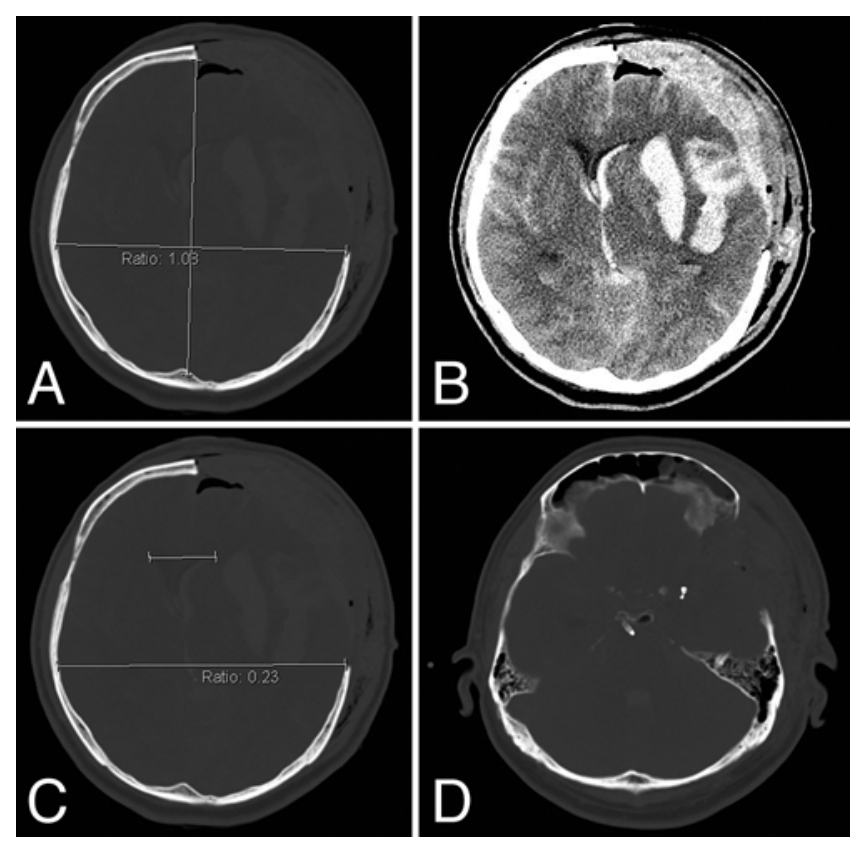

FIG. 3. Axial CT examples of a Kakarla grade 3 patient with a decreased height-to-width ratio (1.08; A), predominantly unilateral abnormality (B), and decreased Evan's index $(0.23 ; \mathbf{C})$, resulting in shift of the normal expected ventricle location. The tip of the EVD catheter is positioned within the interpeduncular cistern (D).

cantly higher rates of both suboptimal and unsatisfactory placements in patients with smaller height-to-width ratios $(\mathrm{p}=0.012$ and $\mathrm{p}=0.003$, respectively). This ratio has not been evaluated in previous literature, although it would further support the hypothesis that altering relative lateral ventricle position in relation to external landmarks results in increased risk for malpositioning during freehand insertion, a technique dependent on relative external landmarks.

\section{Alternative Insertion Techniques}

Several promising alternative EVD placement methods have been proposed in recent years including a guiding fixed tripod, ${ }^{5}$ endoscopic and robotic procedures, ${ }^{12,21}$ bedside neuronavigation procedures, ${ }^{15}$ flat detector CT and live fluoroscopic guidance, ${ }^{3} \mathrm{CT}$ guidance, ${ }^{10}$ and realtime ultrasound guidance through a single burr hole., ${ }^{2,20}$ Concerns regarding efficiency, cost, availability, ease of use, and other modality-specific limitations have restricted widespread use of these alternative techniques. ${ }^{8}$ In a survey, most neurosurgical trainees and staff stated they would not accept more than a 5-minute increase in procedural time for $100 \%$ accuracy. ${ }^{14}$ Interestingly, Krötz et al. has shown that CT-controlled ventriculostomy placement results in significantly shorter $\mathrm{CT}$ to intervention and CT to ICU transfer times when performed in patients presenting to the emergency department, arguing against an increased time requirement for this alternative technique. ${ }^{10}$ Nonetheless, the combination of limiting factors with alternative insertion techniques relative to freehand insertion will likely result in freehand insertion remaining as the preferred technique in the foreseeable future. 
Therefore, stratifying patients at increased risk for suboptimal and unsatisfactory positioning with the freehand technique will assist in identifying patients likely to benefit from an alternative technique.

\section{Limitations and Future Direction}

Our study contains inherent limitations of a singlecenter retrospective study evaluated using a univariate analysis. Although we suspect interrelationship between the significant predictors, there were insufficient outcomes to allow a multivariate analysis. This challenge is also reflected in previous papers by Kakarla et al..$^{9}$ and Toma et al.,22 underscoring the need for prospective multicenter studies. Furthermore, our 2-year review period was chosen based on accessibility of data from our neurosciences ICU rather than a formal power analysis. Although our study is likely sufficiently powered to detect important differences between treatment groups in a univariate analysis, it is possible that we have insufficient power to detect small but clinically important differences. Data were also collected by one author and may be subject to measurement bias. In addition, our radiographic factors were selected on the basis of easily obtainable measurements, and more-involved measurements, such as volume of lateral ventricles, were not evaluated. Focusing on rates of suboptimal and unsatisfactory positioning, we did not assess the number of passes required with each case prior to obtaining the postprocedural CT. We also did not assess associated complications or outcomes directly, such as rates of hemorrhage, infection rates, or failure rates between groups, so we cannot directly ascribe rates of malpositioning with frequency of additional complications. Prospective validation of radiographic factors that indicate relative shift of the ventricles in relation to surface landmarks would more accurately evaluate these measurements' ability to determine rates of malpositioning. Also, as additional techniques for EVD insertion become more widely available and utilized, reevaluating the impact of integrating these predictors into a therapeutic algorithm on the overall rates of malpositioning and clinical outcomes would be valuable.

\section{Conclusions}

Freehand EVD insertion is associated with significant malpositioning into suboptimal functional spaces, cortex, and nontarget CSF spaces. Our study indicates that the likelihood of malpositioning can be predicted with baseline clinical and radiographic features. Specifically, we have identified the patient's head height-to-width ratio as a novel and potentially important radiographic predictor for malpositioning. Through stratifying patients at higher risk for EVD malpositioning, the selective use of alternative/adjunctive placement techniques may improve accuracy rates.

\section{Acknowledgments}

We would like to thank Dr. Derek Emery for his support in arranging for statistical analysis, Mr. Imran Hassan and the EPICORE Center for performing additional statistical analysis, and Ms. Becky Wong and Ms. Kirti Dattani for facilitating patient identification and collection of demographic information from the retrospective study period.

\section{References}

1. Abdoh MG, Bekaert O, Hodel J, Diarra SM, Le Guerinel C, Nseir R, et al: Accuracy of external ventricular drainage catheter placement. Acta Neurochir (Wien) 154:153-159, 2012

2. Coulson NK, Chiarelli PA, Su DK, Chang JJ, MacConaghy B, Murthy R, et al: Ultrasound stylet for non-image-guided ventricular catheterization. J Neurosurg Pediatr 16:393401, 2015

3. Fiorella D, Peeling L, Denice CM, Sarmiento M, Woo $\mathrm{HH}$ : Integrated flat detector CT and live fluoroscopicguided external ventricular drain placement within the neuroangiography suite. J Neurointerv Surg 6:457-460, 2014

4. Gautschi OP, Smoll NR, Kotowski M, Schatlo B, Tosic M, Stimec B, et al: Non-assisted versus neuro-navigated and XperCT-guided external ventricular catheter placement: a comparative cadaver study. Acta Neurochir (Wien) 156:777-785, 2014

5. Ghajar JBG: A guide for ventricular catheter placement. Technical note. J Neurosurg 63:985-986, 1985

6. Hayhurst C, Beems T, Jenkinson MD, Byrne P, Clark S, Kandasamy J, et al: Effect of electromagnetic-navigated shunt placement on failure rates: a prospective multicenter study. $\mathbf{J}$ Neurosurg 113:1273-1278, 2010

7. Hsieh CT, Chen GJ, Ma HI, Chang CF, Cheng CM, Su YH, et al: The misplacement of external ventricular drain by freehand method in emergent neurosurgery. Acta Neurol Belg 111:22-28, 2011

8. Huyette DR, Turnbow BJ, Kaufman C, Vaslow DF, Whiting BB, Oh MY: Accuracy of the freehand pass technique for ventriculostomy catheter placement: retrospective assessment using computed tomography scans. J Neurosurg 108:88-91, 2008

9. Kakarla UK, Kim LJ, Chang SW, Theodore N, Spetzler RF: Safety and accuracy of bedside external ventricular drain placement. Neurosurgery 63 (1 Suppl 1):ONS162-ONS167, 2008

10. Krötz M, Linsenmaier U, Kanz KG, Pfeifer KJ, Mutschler W, Reiser M: Evaluation of minimally invasive percutaneous CT-controlled ventriculostomy in patients with severe head trauma. Eur Radiol 14:227-233, 2004

11. Levitt MR, O'Neill BR, Ishak GE, Khanna PC, Temkin NR, Ellenbogen RG, et al: Image-guided cerebrospinal fluid shunting in children: catheter accuracy and shunt survival. J Neurosurg Pediatr 10:112-117, 2012

12. Lollis SS, Roberts DW: Robotic catheter ventriculostomy: feasibility, efficacy, and implication. J Neurosurg 108:269274, 2008

13. O'Leary ST, Kole MK, Hoover DA, Hysell SE, Thomas A, Shaffrey CI: Efficacy of the Ghajar Guide revisited: a prospective study. J Neurosurg 92:801-803, 2000

14. O'Neill BR, Velez DA, Braxton EE, Whiting D, Oh MY: A survey of ventriculostomy and intracranial pressure monitor placement practices. Surg Neurol 70:268-273, 2008

15. Patil V, Gupta R, San José Estépar R, Lacson R, Cheung A, Wong JM, et al: Smart stylet: the development and use of a bedside external ventricular drain image-guidance system. Stereotact Funct Neurosurg 93:50-58, 2015

16. Phillips SB, Delly F, Nelson C, Krishnamurthy S: Bedside external ventricular drain placement: can multiple passes be predicted on the computed tomography scan before the procedure? World Neurosurg 82:739-744, 2014

17. Roberts DW: Is good good enough? Neurocrit Care 10:155156, 2009

18. Saladino A, White JB, Wijdicks EFM, Lanzino G: Malplacement of ventricular catheters by neurosurgeons: a single institution experience. Neurocrit Care 10:248-252, 2009 
19. Srinivasan VM, O'Neill BR, Jho D, Whiting DM, Oh MY: The history of external ventricular drainage. J Neurosurg 120:228-236, 2014

20. Strowitzki M, Komenda Y, Eymann R, Steudel WI: Accuracy of ultrasound-guided puncture of the ventricular system. Childs Nerv Syst 24:65-69, 2008

21. Theodosopoulos PV, Abosch A, McDermott MW: Intraoperative fiber-optic endoscopy for ventricular catheter insertion. Can J Neurol Sci 28:56-60, 2001

22. Toma AK, Camp S, Watkins LD, Grieve J, Kitchen ND: External ventricular drain insertion accuracy: is there a need for change in practice? Neurosurgery 65:1197-1201, 2009

23. Wan KR, Toy JA, Wolfe R, Danks A: Factors affecting the accuracy of ventricular catheter placement. J Clin Neurosci 18:485-488, 2011

24. Wilson TJ, Stetler WR Jr, Al-Holou WN, Sullivan SE: Comparison of the accuracy of ventricular catheter placement using freehand placement, ultrasonic guidance, and stereotactic neuronavigation. J Neurosurg 119:66-70, 2013

25. Woernle CM, Burkhardt JK, Bellut D, Krayenbuehl N, Bertalanffy H: Do iatrogenic factors bias the placement of external ventricular catheters? A single institute experience and review of the literature. Neurol Med Chir (Tokyo) 51:180-186, 2011

\section{Disclosures}

Dr. O'Kelly reports serving as a proctor and course instructor for Pipeline stents (Medtronic).

\section{Author Contributions}

Conception and design: Wilson, Rempel. Acquisition of data: Wilson. Analysis and interpretation of data: Wilson, O'Kelly. Drafting the article: Wilson, O'Kelly, Jack. Critically revising the article: all authors. Reviewed submitted version of manuscript: all authors. Approved the final version of the manuscript on behalf of all authors: Wilson. Statistical analysis: O'Kelly.

\section{Supplemental Information}

Previous Presentations

Portions of this work were previously presented at the Canadian Association of Radiologists Annual Meeting in 2017 and at the American Society of Neuroradiology Annual Meeting in 2017.

\section{Correspondence}

Mitchell P. Wilson: University of Alberta, Edmonton, AB, Canada.mitch.wilson@ualberta.ca. 